Комментарий к статье

Р.С. Лакотко и соавт.

«Риск и значимость венозной

воздушной эмболии

при нейрохирургических

операциях в положении сидя

у взрослых»

А.Ю. Лубнин

ФГАУ «НМИЦ нейрохирургии им. акад. Н.Н. Бурденко» МЗ РФ, Москва, Россия

凶 Для корреспонденции: Лубнин Андрей Юрьевич д-р мед. наук, профессор, руководитель отдела анестезиологии, реанимации и интенсивной терапии ФГАУ «НМИЦ нейрохирургии им. акад. Н.Н. Бурденко» МЗ РФ, Москва; e-mail: lubnin@nsi.ru

匹 Для цитирования: Лубнин А.Ю. Комментарий к статье Р.С. Лакотко и соавт. «Риск и значимость венозной воздушной эмболии при нейрохирургических операциях в положении сидя у взрослых». Вестник интенсивной терапии им. А.И. Салтанова. 2020;1:100-104.

« Поступила: 28.12.2019

目 Принята к печати: 05.11.2019
Comment on the article of R.S. Lakotko et al. "Risk and significance of venous air embolism at neurosurgical operations in the position of sitting at adults"

A.Yu. Lubnin

Burdenko National Neurosurgery Center, Moscow, Russia

$\otimes$ For correspondence: Lubnin Yu. Andrey - Dr. Med. Sci, professor, Burdenko National Neurosurgery Center, Moscow; e-mail: lubnin@nsi.ru

ए For citation: Lubnin A.Yu. Comment on the article of R.S. Lakotko, et al. "Risk and significance of venous air embolism at neurosurgical operations in the position of sitting at adults". Annals of Critical Care. 2020;1:100-104.

匹 Received: 28.12.2019

自 Accepted: 05.11.2019
Венозная воздушная эмболия (ВВЭ) и ее вторичные осложнения, такие как парадоксальная воздушная эмболия (ПВВЭ) и респираторный дистресс-синдром, бесспорно представляют собой для нейрохирургических пациентов, оперируемых в положении сидя, тяжелые интраоперационные осложнения, потенциально способные привести не только к инвалидизации, но и к гибели больного. Эти клинические ситуации неоднократно описаны в литературе и, к сожалению, имели место и в нашей практике. Это одна из актуальных тем в современной нейроанестезиологии, и поэтому, на мой взгляд, необходимо поблагодарить авторов за то, что они обратились к этой проблеме [1]. Дизайн исследования выглядит вполне адекватным. Однако количество обследованных больных - 66 пациентов за 3,5 года невелико (в нашей клинике, пока мы не пересмотрели своего отношения к операциям в положении сидя, в год оперировалось 1000-1500 таких больных), ну, сколько уж получилось. Для ранней диагностики ВВЭ авторы статьи используют самую чувствительную модальность - транспищеводную эхокардиографию. Такой подход позволяет авторам диагностировать факт развития ВВЭ в 52 \% наблюдений, что, на мой взгляд, не так уж и мало (хотя в литературе есть и более высокие цифры - до 80 \%), а вот последствия ВВЭ оцениваются на основании простого клинического анализа, что, вполне естественно, не позволяет выявить каких-либо последствий осложнения. На основании полученных 
данных авторы делают достаточно категоричный вывод, который наиболее жестко звучит в конце резюме: «...Модель анестезиологического обеспечения, подобная нашей, эффективна в предотвращении основных осложнений, связанных с положением пациента сидя. Страх перед фатальными осложнениями представляется необоснованным». Я занимаюсь проблемой ВВЭ достаточно давно (первые мои публикации на эту тему относятся к 1991 г. [2, 3]), имею собственный многолетний клинический опыт в этом отношении, и, видимо, поэтому мне сложно согласиться с выводами авторов этой статьи. Попробую объяснить почему.

1. Патофизиология ВВЭ. История изучения ВВЭ насчитывает не одно столетие [4], она включает и экспериментальные, и клинические исследования, и сейчас мы вправе сказать, что патофизиология ВВЭ и ПВВЭ изучена достаточно полно. Повреждение стенки венозного коллектора в ходе нейрохирургической операции (практически неустранимая причина) создает главное условие для поступления атмосферного воздуха в просвет сосуда (главным компонентом атмосферного воздуха является азот - плохо диффундирующий газ). Далее с током крови воздушный эмбол достигает правых отделов сердца, где в случае массивной эмболии возникает ситуация т. н. воздушного замка, приводящая к резкому снижению выброса крови в легочную артерию. Это проявляется снижением значений $\mathrm{EtCO}_{2}$, АД и $\mathrm{SpO}_{2}$. В тяжелых случаях развивается асистолия. При дальнейшем прохождении воздушных эмболов в систему легочной артерии возможно блокирование воздушными эмболами микроциркуляторного русла с последующим присоединением тромбоза и формированием респираторного дистресс-синдрома [5].

При ПВВЭ воздушные эмболы попадают в левые отделы сердца и большой круг кровообращения, приводя к эмболии церебральных и коронарных артерий. Поступление воздушных эмболов в большой круг кровообращения возможно двумя путями: через септальные дефекты в сердце и по анастомозам между ветвями легочной и бронхиальных артерий. Это клинически важная информация. Во-первых, частота септальных дефектов, как оказалось, достаточно высока в популяции, и они могут себя клинически никак не проявлять [6]. Авторы статьи приводят свою частоту в 36 \%, причем это только овальное окно. Это неожиданно высокая цифра. Много лет назад в США было проведено простое исследование. Почти 1000 умерших по различным причинам детей были целенаправленно исследованы при вскрытии на предмет наличия септальных дефектов в сердце. Частота обнаружения только овального отверстия составила тоже немалую цифру - $25 \%$, но все же это не 36 \%. Вряд ли наличие нейрохирургической патологии предрасполагает к более высокой частоте септальных дефектов в сердце, поэтому, как мне кажется, авторам необходимо проверить свои данные с помощью других методов. Наличие относительно высокого процента септальных дефектов, конечно же, возбудило нейроанестезиологов. Сначала было предложено всем больным, которым планируется проведение нейрохирургического вмешательства в положении сидя, в обязательном порядке проводить до операции контрастную эхокардиографию и в случае обнаружения функционирующего овального отверстия таких пациентов сидя ни в коем случае не оперировать. Позднее было предложено более эффективное решение - проводить этим пациентам до нейрохирургического вмешательства эндоваскулярную окклюзию септального дефекта [7]. Но более важным мне представляется другой факт - наличие сосудистых анастомозов, по которым воздушные эмболы могут попадать в сосуды большого круга кровообращения. Сначала это были клинические наблюдения отдельных больных, которые погибали от ПВВЭ, но у которых на секции септальных дефектов обнаружить не удавалось (например, Singbartl et al. [8]). Но были и результаты экспериментов на лабораторных животных, убедительно доказавшие принципиальную возможность такого перехода воздушных эмболов [9] и интраоперационные регистрации пассажа воздушных эмболов справа налево с помощью транспищеводной эхокардиографии [10]. Затем появились работы, в которых было показано, что некоторые фармакологические препараты, и в особенности ингаляционные анестетики (!), способствуют такому переходу воздушных эмболов (в схеме анестезии фигурировали галотан и изофлуран $[4,11])$. Все это существенная информация, но, на мой взгляд, наиболее важным следствием наличия феномена пассажа воздушных эмболов является то, что отсутствие септального дефекта или его предварительное хирургическое закрытие не гарантирует устранение риска развития ПВВЭ. В этой связи все, что авторы статьи написали по поводу обнаружения септального дефекта и отказа от использования ПДКВ (об этом методе отдельно ниже), особого смысла не имеет.

Последнее, что мне хотелось бы отметить по патофизиологии ВВЭ, - это три фактора ее развития у нейрохирургических больных, оперируемых в положении сидя, что имеет непосредственное отношение к профилактике ВВЭ: 1) практически неизбежное повреждение венозных коллекторов черепа и мозга во время трепанации (неустранимый фактор); 2) наличие ригидных стенок церебральных вен, и в особенности синусов мозга, которые препятствуют их спадению в случае их ранения и развития отрицательного давления в них (в отличие от мягкостенных периферических вен, которые в этой ситуации просто спадаются); 3) отрицательное давление по отношению к атмосферному в венозных коллекторах черепа и мозга вследствие ряда причин, таких как операционная кровопотеря, исходная гиповолемия и постуральные реакции кровообращения. Обо всем этом мы тоже давно писали [12]. 
2. Диагностика ВВЭ. Авторы статьи для диагностики ВВЭ использовали транспищеводную эхокардиографию и другие общепринятые методы $\left(\mathrm{EtCO}_{2}\right.$, $\mathrm{SpO}_{2}$ и инвазивное АД). Доказано, что транспищеводная эхокардиография обладает исключительно высокой чувствительностью в диагностике ВВЭ, позволяя обнаруживать мельчайшие воздушные эмболы объемом 0,01 мл [4]. Однако даже этот высокочувствительный метод констатирует уже развившееся осложнение. Он позволяет анестезиологу быстро отреагировать на развитие осложнения, но даже первоначальное поступление воздуха может быть столь массивным, что вызовет асистолию. Далее алгоритм действий анестезиолога прост: компрессия шейных вен для обнаружения источника поступления воздуха - исключительно простой и эффективный метод, приводящий также к прекращению дальнейшего поступления воздуха в сосуд. Это, конечно, не только диагностика, но уже терапия осложнения. Хотя авторы статьи считают такой маневр только диагностическим. Приводимые ими пояснения по поводу оттока крови из полости черепа в положении сидя в сторону венозных сплетений спинного мозга представляются достаточно умозрительными.

3. Терапия ВВЭ. Для ситуации массивной ВВЭ она разработана. Это прежде всего непрямой массаж сердца, который был предложен Ericsson et al. еще в 1964 г. [13]. Действительно, несколько массажных движений разбивают большой воздушный пузырь на мелкие пузырьки, которые затем с током крови выбрасываются в легочную артерию. Так как суммарная площадь сосудистого русла легочной артерии огромна, то чаще всего это не приводит к каким-либо серьезным последствиям. У трех пациентов в обсуждаемой статье развилась клиническая симптоматика ВВЭ. К сожалению, из текста статьи я так и не понял, использовали авторы непрямой массаж сердца у этих больных или нет. Дальнейшая терапия включает в себя переход на $100 \% \mathrm{O}_{2}$, форсированную внутривенную инфузию коллоидов, гормоны и вазопрессоры по необходимости и, конечно, максимально оперативное закрытие дефекта в стенке венозного коллектора любым доступным способом. Из текста обсуждаемой работы остается непонятным, какие действия предпринимали авторы в случае констатации факта развития ВВЭ, в частности, у этих трех пациентов с клиническими симптомами ВВЭ.

Предложенный Bunegin и Albin метод эвакуации воздуха из правых отделов сердца через заранее установленный туда специальный многопросветный катетер показал себя эффективным в эксперименте [14], но клинически оказался достаточно трудоемким и поэтому не получил широкого распространения.

4. Профилактика ВВЭ. Для целей профилактики ВВЭ у нейрохирургических больных, оперируемых в положении сидя, предложено несколько методов, направленных на повышение давления в синусах мозга выше атмосферного (это единственная из причин ВВЭ, на которую мы можем хоть как-то реально повлиять). Вот они: 1) надувной шейный турникет; 2) управляемая умеренная гиперкапния; 3) положительное давление в конце выдоха (ПДКВ) - о которых мы также давно писали $[15,16]$. Эффективность первых двух методов доказана клинически с помощью непрерывной регистрации величины внутрисинусного давления. Метод ПДКВ, наоборот, показал свою неэффективность, а в эксперименте он увеличивал частоту гемодинамической нестабильности и ПВВЭ за счет формирования градиента давлений [7]. Другие методы, такие как высокое поднятие ножного конца операционного стола, военные противошоковые брюки, внутривенная волемическая нагрузка коллоидными растворами в начале операции, доказанной эффективности не имеют и могут рассматриваться только как вспомогательные. Из текста обсуждаемой работы в отношении использованных авторами методов профилактики ВВЭ не понятно, использовали они что-либо или нет.

5. Главное. По результатам своего достаточно ограниченного исследования авторы считают страх перед развитием фатальных осложнений ВВЭ необоснованным. И это то главное, с чем я никак согласиться не могу по ряду серьезных причин. Во-первых, тяжелые осложнения ВВЭ реально есть, они описаны в литературе, и, увы, мы сами сталкивались с ними [17]. Если авторы статьи у своих 66 пациентов с этим не столкнулись, то и слава богу. Но, когда количество пациентов увеличится до 600 или 6000 , боюсь, статистика осложнений неизбежно станет другой. Во-вторых, еще в 2013 г. в журнале «Анестезиология и реаниматология» мы опубликовали результаты нашего исследования [18], которое достаточно странно цитируют авторы обсуждаемой статьи. На предмет осложнений и прежде всего ВВЭ были сравнены две группы нейрохирургических больных с патологией задней черепной ямки, по 100 пациентов в каждой группе, оперированных лежа и сидя соответственно [18]. Частота развития ВВЭ (диагностирована с помощью транспищеводного и прекордиального допплера) в группе пациентов, оперированных сидя, была в 28 раз (!!!) выше, чем в группе оперированных лежа. Мне кажется, к этому сложно что-то добавить. Отказ от проведения хирургических вмешательств в положении сидя, по-видимому, и является самой эффективной профилактикой ВВЭ у нейрохирургических больных.

В-третьих, и это мне представляется особенно грустным, анализ серии публикаций последнего времени свидетельствует о какой-то малопонятной «рас- 
слабленности» в отношении ВВЭ в положении сидя в нейрохирургии. Кто-то изобретает новую терминологию: вместо положения сидя - «положение полусидя» или «положение молящегося», кто-то считает, что угол наклона самой головы в положении сидя не в 60, а в 30 градусов может что-то серьезно изменить [19-22]. Но, глядя на фото пациентов из этих работ, отчетливо видно, что это и есть самое настоящее положение сидя. Кто-то поднимает ноги пациента к потолку в надежде, что это спасет от ВВЭ. Но чаще отмечается игнорирование проблемы, потому что «серьезных осложнений немного и от них редко погибают» $[16,21,23-25]$. Все как у авторов обсуждаемой работы. А я вот хорошо помню всех наших больных, погибших от ВВЭ и ее вторичных осложнений, и эта цена за удобство оперирования мне кажется необоснованно высокой. Это удобство того стоит? Не уверен.

Конфликт интересов. Автор заявляет об отсутствии конфликта интересов.

Вклад автора. Лубнин А.Ю. - написание статьи.

\section{ORCID aвTOPa}

Лубнин А.Ю. - 0000-0003-2595-5877

\section{Литература/References}

[1] ЛакоткоР.С., Аверьянов Д.А., ЩеголевА.В.Риски значимость венозной воздушной эмболии при нейрохирургических операциях в положении сидя у взрослых. Вестник интенсивной терапии им. А.И. Салтанова. 2019; 4: 68-72. DOI: 10.21320/1818-474X-2019-4-68-72

[Lakotko R.S., Averyanov D.A., Shchyogolev A.V. Risk and significance of venous air embolism at neurosurgical operations in the position of sitting at adults. Article. Annals of Critical Care. 2019; 4:68-72. (In Russ)]

[2] Лубнин А.Ю. Повторная массивная воздушная эмболия у пациента, оперированного в положении сидя. Анестезиология и реаниматология. 1991; 4: 36-37.

[Lubnin A.Yu. Povtornaya massivnaya vozdushnaya emboliya u pacienta, operirovannogo $v$ polozhenii sidya. Anesteziologiya i reanimatologiya. 1991; 4: 36-37. (In Russ)]

[3] Лубнин А.Ю., Ананов М.В. Массивная воздушная эмболия как осложнение при хирургической коррекции сагиттального краниостеноза. Вопросы нейрохирургии им. Н.Н. Бурденко. 1992; 5: 36-38.

[Lubnin A.Yu., Ananov M.V. Massivnaya vozdushnaya emboliya kak oslozhnenie pri hirurgicheskoj korrekcii sagittal?nnogo kraniostenoza. Problems of neurosurgery named after N.N. Burdenko. 1992; 5: 36-38. (In Russ)]

[4] Albin M.S. Air embolism. In: Textbook of Neuroanesthesia and neuroscience perspectives. Ed. M.S. Albin. 1997. NY: McGraw-Hill Co. P. 1009-1026.

[5] Saigal D., Ganjoo P., Tetarway M., Kiro K. Acute pulmonary edema and thrombocytopenia following venous air embolism during sitting position neurosurgery. Asian J. Neurosurg. 2017; 12: 214-216. DOI: $10.4103 / 1793-5482.150223$

[6] Hagen P.T., Scholz D.G., Edwards W.D. Incidence and size of patent foramen ovale during the first 10 decades of life: an autopsy study of 965 normal hearts. Mayo Clin. Proc. 1984; 59: 17-20.
[7] Fathi A.R., Esthehardi P., Mier B. Patent foramen ovale and neurosurgery in sitting position: a systematic review. $\mathrm{Br}$. J. Anaesth. 2009; 102: 588-596.

DOI: 10.1093/bja/aep063

[8] Singbartl G., Metzger G., Stogbauer R., Haarman W. Paradoxe luftembolie bei geschlossenem foramen ovale. Anaesthesist. 1990; 39: 44-49.

[9] Butler B.D., Hills B.A. Transpulmonary passage of venous air emboli. J. Appl. Physiol. 1985; 59: 543-547.

[10] Maragkos G.A., Davanzo J., Roberts S.M., et al. Paradoxical Air Embolism Without Patent Foramen Ovale During Craniotomy in the Sitting Position. Cureus. 2019; 11(4): e4355. DOI: 10.7759/cureus.4355

[11] Booke M., Bone H.G., VanAken H., et al. Die Venose paradoxe Luftembolie. Anaesthesist. 1999; 48: 236-241.

[12] Лубнин А.Ю., Осканова М.Ю. Воздушная эмболия при нейрохирургическихвмешательствах. Вопросынейрохирургии им. Н.Н. Бурденко. 1995; 4: 60-68.

[Lubnin A.Yu., Oskanova M.Yu. Vozdushnaya emboliya prinejrohirurgicheskih vmeshatel[?stvah. Problems of neurosurgery named after N.N. Burdenko. 1995; 4: 60-68. (In Russ)]

[13] Ericsson J.A., Gottlieb J.D., Sweet R.B. Closed-chest cardiac massage in the treatment of venous air embolism. N. Engl. J. Med. 1964; 270: 1353-1354.

[14] Colley P.S., Artru A.A. Bunegin-Albin catheter improves air retrieval and resuscitation from lethal venous air embolism in dogs. Anesth. Analg. 1987; 66: 991-994.

[15] Лубнин А.Ю., Осканова М.Ю. Профилактика воздушной эмболии у нейрохирургических больных, оперируемых в положении сидя: сравнительное изучение трех методов. Анестезиология и реаниматология. 1994; 5: 22-25.

[Lubnin A.Yu., Oskanova M.Yu. Profilaktika vozdushnoj embolii u nejrohirurgicheskih bol'nyh, operiruemyh $v$ polozhenii sidya: sravnitel'noe izuchenie trekh metodov. Anesteziologiya i reanimatologiya. 1994; 5: 22-25. (In Russ)]

[16] Лубнин А.Ю., Лукьянов В.И., Осканова М.Ю. Профилактика воздушной эмболии у нейрохирургических больных, оперируемых в положении сидя: Метод управляемого 
внутрисинусного давления. Вопросы нейрохирургии им. Н.Н. Бурденко. 1994; 4: 14-17.

[Lubnin A.Yu., Luk'yanov V.I., Oskanova M.Yu. Profilaktika vozdushnoj embolii u nejrohirurgicheskih bolannyh, operiruemyh $v$ polozhenii sidya: Metod upravlyaemogo vnutrisinusnogo davleniya. Problems of neurosurgery named after N.N. Burdenko. 1994; 4: 14-17. (In Russ)]

[17] Ананьев Е.П., Полупан А.А., Савин И.А. и др. Парадоксальная воздушная эмболия, приведшая к острому инфаркту миокарда и массивному ишемическому повреждению мозга у пациента, оперированного в положении сидя. Вопросы нейрохирургии им. Н.Н. Бурденко. 2016; 2: 77-84. DOI: $10.17116 /$ neiro201680284-92

[Anan'ev E.P., Polupan A.A, Savin I.A. et al. Paradoxical air embolism resulted in acute myocardial infarction and massive ischemic brain injury in a patient operated on in a sitting position. Problems of neurosurgery named after N.N. Burdenko. 2016; 2: 77-84. (In Russ)]

[18] Исраелян Л.А., Шиманский В.Н., Одаманов Д.А. u др. Положение больного на операционном столе в нейрохирургии: сидя или лежа. Анестезиология и реаниматология. 2013; 4: 18-26.

[Israelyan L.A., Shimanskiy V.N., Otamanov D.A. et al. Patient positioning on the operating table in neurosurgery: sitting or lying. Anesteziologiya i Reanimatologiya. 2013; 4: 18-26. (In Russ)]

[19] Choque-Velasquez J., Colasanti R., Rezendiz-Nieves J.C., et al. Venous air emdolism and sitting position in Helsinki pineal region surgery. Surg. Neurol. Int. 2018; 9: 160.

DOI: 10.4103/sni.sni_128_18
[20] Feigl G.C., Decker K., Wurms M., et al. Neurosurgical procedures in the semisitting position: Evaluation of the risk of paradoxical venous air embolism in patients with patent foramen ovale. World Neurosurg. 2014; 12: 739-744. DOI: 10.1016/j.wneu.2013.01.003

[21] Klein J., Jurati T.A., Weise M., et al. A systematic review of the semisitting position in neurosurgical patients with patent foramen ovale: How frequent is paradoxical embolism? World Neurosurg. 2018; 16: 198-200.

DOI: 10.1016/j.wneu.2018.04.114

[22] Ture H., Harput M.W., Bekiroglu N., et al. Effect of the degree of head elevation on the incidence and severity of venous air embolism in cranial neurosurgical procedures with patients in the semisitting position. J. Neurosurg. 2018; 128(5): 1560-1569. DOI: 10.3171/2017.1.JNS162489

[23] Gracial., Fabregas N. Craniotomy in sitting position: Anesthesiology management. Curr. Opin. Anesthesiol. 2014; 27: 474-483. DOI: $10.1097 / A C O .0000000000000104$

[24] Himes B.T., Mallory G.W., Absejo A., et al. Contemporary analysis of intraoperative and perioperative complications of neurosurgical procedures performing in the sitting position. J. Neurosurg. 2017; 127: 182-188. DOI: 10.3171/2016.5.JNS152328

[25] Dallier F.D., Di Roio C. Sitting position for pineal surgery: Some anesthetic considerations. Neurochirurgie. 2015; 61: 164-167. DOI: 10.1016/j.neurochi.2014.10.110 